\title{
CRISPR/Cas9-mediated genome editing induces exon skipping by complete or stochastic altering splicing in the migratory locust
}

\author{
Dafeng Chen ${ }^{1,3+}$, Ji-Xin Tang ${ }^{2 \dagger}$, Beibei Li', Li Hou' ${ }^{1}$ Xianhui Wang ${ }^{1,3^{*}}$ and Le Kang ${ }^{1,3^{*}}$
}

\begin{abstract}
Background: The CRISPR/Cas9 system has been widely used to generate gene knockout/knockin models by inducing frameshift mutants in cell lines and organisms. Several recent studies have reported that such mutants can lead to in-frame exon skipping in cell lines. However, there was little research about post-transcriptional effect of CRISPR-mediated gene editing in vivo.

Results: We showed that frameshift indels also induced complete or stochastic exon skipping by deleting different regions to influence pre-mRNA splicing in vivo. In the migratory locust, the missing 55 bp at the boundary of intron 3 and exon 4 of an olfactory receptor gene, LmigOr35, resulted in complete exon 4 skipping, whereas the lacking 22 bp in exon 4 of LmigOr35 only resulted in stochastic exon 4 skipping. A single sgRNA induced small insertions or deletions at the boundary of intron and exon to disrupt the 3' splicing site causing completely exon skipping, or alternatively induce small insertions or deletions in the exon to stochastic alter splicing causing the stochastic exon skipping.

Conclusions: These results indicated that complete or stochastic exon skipping could result from the CRISPRmediated genome editing by deleting different regions of the gene. Although exon skipping caused by CRISPRmediated editing was an unexpected outcome, this finding could be developed as a technology to investigate pre-mRNA splicing or to cure several human diseases caused by splicing mutations.
\end{abstract}

Keywords: CRISPR/Cas9, Gene editing, Exon skipping, Migratory locust, Pre-mRNA splicing

\section{Background}

Clustered Regularly Interspaced Short Palindromic Repeats/CRISPR associated protein 9 (CRISPR/Cas9), which is a rapid and efficient system to generate genome mutation, has been used in many organisms for gene function loss [1-9]. Short frameshift insertion-deletions (indels) are usually introduced in exonic sequences to disrupt the reading frame of mRNA by CRISPR/Cas9. These indels are created by an endogenous DNA repair machinery via non-homologous end joining (NHEJ) when the Cas9

\footnotetext{
* Correspondence: wangxh@ioz.ac.cn; lkang@ioz.ac.cn

${ }^{\dagger}$ Dafeng Chen and Ji-Xin Tang contributed equally to this work.

${ }^{1}$ State Key Laboratory of Integrated Management of Pest Insects and

Rodents, Institute of Zoology, Chinese Academy of Sciences, Beijing 100101,

China

Full list of author information is available at the end of the article
}

nuclease generates double-strand breaks (DSB). Currently, many studies have focused on frameshift mutation by generating DSB for genome editing purposes. One major application of CRISPR/Cas9 system is to generate inactivating mutations in protein-coding genes by targeting single sgRNA sites to create frameshifts. Most of the indels in protein-coding gene exons are supposed to be frameshift mutations disrupting open reading frames with the obvious exception of those whose size is multiple of three. Frameshift indels are very suitable for generating loss-of-function mutations in protein coding genes. These mutated transcripts are recognized and degraded by a nonsense-mediated mRNA decay (NMD) machinery or are translated into truncated non-functional proteins. 
Pre-mRNA splicing is catalyzed by the spliceosome, one of the largest ribonucleoproteic complex of the cell. Through splicing intronic sequences of pre-mRNA are eliminated from pre-mRNA and exonnic sequences are joined together. Pre-mRNA splicing requires several cis-acting elements on the pre-mRNA: (1) $5^{\prime}$ donor and 3 ' acceptor splice site consensus sequences, by which the exon-intron boundaries are constituted; (2) a branch point, which is consisted by an adenosine, located in a consensus sequence of the intron, 18-40 nucleotides upstream of the $3^{\prime}$ acceptor splice site [10]. The $5^{\prime}$ and $3^{\prime}$ splice site and branch point is essential for the pre-mRNA splice, therefore they should be considered when we generated the frame-shift mutant by CRISPR/ Cas9 system.

Recently, several studies have reported the unintended consequences at the post-transcriptional level, such as aberrant RNA splicing, caused by CRISPR-mediated editing of the target gene [11]. The insertion of a large DNA fragment into an exon of human $h C D C 14 A$ and $h C D C 14 B$ genes by genome editing introduced its skipping from the final transcript in human hTERT-RPE1 and HCT116 cells [12]. A single base change in the target exon of human FLOT-1 gene resulted in random splicing in HeLa cells [13]. The frameshift indels engineered by CRISPR/Cas9 also led to skipping of "multiple three nucleotides" [14]. A single sgRNA induced partial exon splicing or unexpected large deletions that removed exons [15]. These in vitro studies on cell lines revealed other artifactual effects of CRISPR applications aside from their off-target effects, thereby providing new information for better mutant allele screening. Recent study in zebrafish showed that disrupted ESE by inserted $7 \mathrm{bp}$ nucleotides could resulted into exon skipping [16]. These results suggest that stochastic exon skipped induced by the indels in exon can be found both in vivo and in vitro. However, few of in vivo studies have been designed specifically to disrupt the cis-acting elements (such as the $5^{\prime}$ and $3^{\prime}$ splice sites) by CRISPR-mediated genome editing due to the complexity of the cis-acting elements during the pre-mRNA splicing in organisms [17]. Besides the $5^{\prime}$ and $3^{\prime}$ splice sites there are many cis-acting elements (such as splicing enhancers or silencers in exons or in introns) that can influence the pre-mRNA splicing. Therefore, it is difficult to identify the cis-acting elements and to disrupt them precisely by genome editing.

The CRISPR/Cas9 system has been successfully applied to generate knockout mutant lines in the migratory locust, Locusta migratoria [7], which has served as model species for phenotypical plasticity involved in behavior, morphology, and physiology [18-24]. The genome sequencing of locust showed that this insect had a huge genome $(6.5 \mathrm{~Gb})$ and displayed the unique characteristics on the splicing mechanisms of long introns compared with other insect species [25]. The unique characteristics of locust genome were mainly in proliferation of a diverse range of repetitive elements, the lowest divergent of DNA transposon and big intron and so on [25]. Previous reports have indicated that most insects have an enrichment of ratcheting point sites to allow for efficient splicing of long introns, whereas vertebrates use repetitive elements to aid in splicing long introns and the splicing mechanisms may be convergent evolution associated with the genome size expansion in animals [26]. Probably, locusts is a potential model for studying the effect of gene editing on exon splicing in large genome organisms by CRISPR/Cas9 system.

An olfactory receptor gene suitable for investigating gene editing was selected to perform splicing disruptions in vivo, because the knockout of these olfactory receptors (Ors) was not lethal. Here, we showed that CRISPR-mediated editing of one olfactory receptor gene of locusts induced complete or stochastic exon skipping. Complete exon skipping was caused by the boundary deletion of intron and exon ( 3 ' splice site) that completely altered the pre-mRNA splicing, whereas stochastic exon skipping was due to the alternative splicing that caused by the indels in exon, which changed the cis-effector that promotes the pre-mRNA splicing.

\section{Results \\ Deletion of one 3' splice site of LmigOr35 using CRISPR/ Cas9 system}

In the migratory locust, the repertoire of the Or gene family (142 genes) has recently been identified [27], and LmigOr35 is one of 142 olfactory receptor gene family that only has one transcript and is specifically expressed in the antenna of migratory locust. LmigOr 35 contain 5 exons and four introns (Fig. 1a). We designed the targeted site of Cas9 protein at exon 4 near the 3 ' splicing site (Fig. 1a). We obtained 22 kinds of mutations of LmigOr35 using CRISPR/Cas9 system (Additional file 1: Figure S1). The 55 bp nucleotide missing mutation was one of these mutations (Fig. 1b and Additional file 1: Figure S1). The missed 55 bp nucleotides contained $15 \mathrm{bp} \mathrm{nu}-$ cleotides from the intron 3 of LmigOr35 and $40 \mathrm{bp}$ nucleotides from the exon 4 of LmigOr35 (Fig. 1b). The 55 bp nucleotide deletion caused the missing of 3' splice site at the boundary of intron 3 and exon 4 of LmigOr35 (Fig. 1b). To confirm the wild $(+/+)$ and 55 bp deletion mutants $(-/-)$, we amplified the genome sequences using the primers $\mathrm{F} 1$ and $\mathrm{R} 1$, and then sequenced the PCR products. We found that the 55 bp deletion homozygous mutant $(-/-)$ had a shorter product compared with the wild types (WT) $(+/+)$ (Fig. 1c). The sequencing of PCR products showed that the 55 bp deletion mutation lost the $55 \mathrm{bp}$ nucleotides at the boundary of intron 

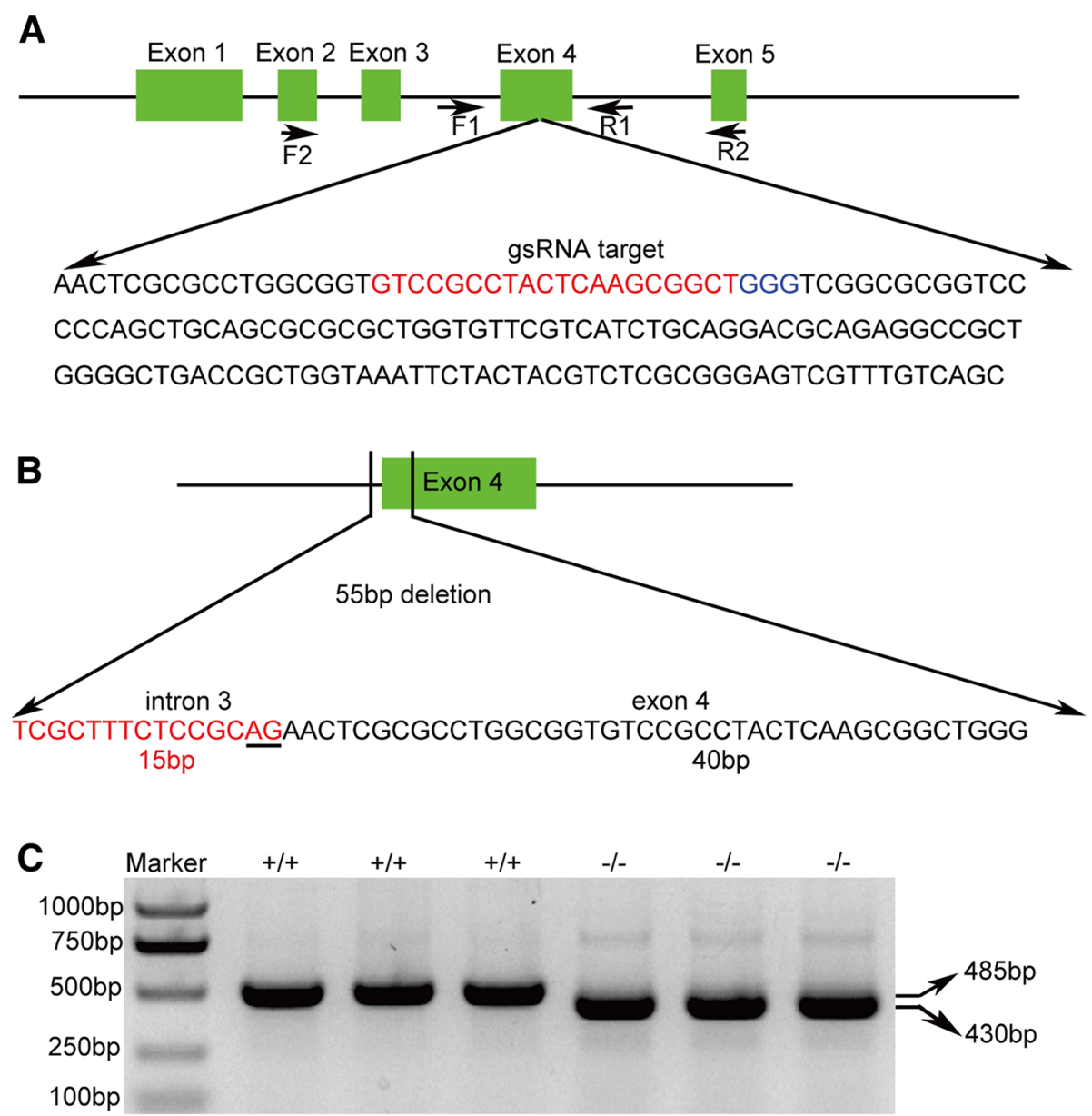

Fig. 1 Disrupting the 3' splice site by deletion of 55 bp nucleotides at the boundary of intron 3 and exon 4 of locust LmigOr35 using CRISPR/ Cas9 system. a The entire gene structure of LmigOr35 with all introns and exons and the designed sgRNA targeted site in exon 4 of locust LmigOr35. F1 and R1 are primers for detecting genome deletion; F2 and R2 are primers for detecting exon deletion. b Deleted nucleotides containing $15 \mathrm{bp}$ intron 3 and 40 bp exon 4 nucleotides of locust LmigOr35. Under line shows the conserved nucleotides in the splice site that are crucial for normal splicing. c Genotype of WT (+/+) and 55 bp mutant (-/-) locusts. The WT locusts obtain a 485 bp brand and the 55 bp mutants obtain a 430 bp brand

3 and exon 4 of $\mathrm{LmigOr} 35$ (Fig. 2). These results indicated that the CRISPR/Cas9 system successfully generated the lacking mutant of 3' splice site in the migratory locust.

\section{Missing 3' splice site at the boundary of intron 3 and exon 4 caused complete exon 4 skipping}

We investigated the consequences when the 3 ' splice site of LmigOr35 in the migratory locust was disrupted. We extracted the total RNA from the antenna of WT and mutation lines of the locusts and reversed the total RNA to be cDNA. We amplified exon 3 to exon 5 of LmigOr35 cDNA. The samples from the WT locusts were a $380 \mathrm{bp}$ band, and the samples from mutant lines only were a $224 \mathrm{bp}$ band. A total of $156 \mathrm{bp}$ losses were observed in the mutant lines and this $156 \mathrm{bp}$ was exactly the sequence of exon 4 (Fig. 3a). A 340 bp band was observed if the mutant samples were normally spliced because 40 bp was missing in exon 4 of the mutant lines. After the sequencing of $224 \mathrm{bp}$ PCR products, we determined that the mutant samples did not contain complete exon 4 compared with the WT samples (Fig. 3b and c). Then, we cloned the PCR products of WT samples and $55 \mathrm{bp}$ mutant determined that all clones in WT samples contained complete exon 4 and no exon 4 was found in all 55 bp mutants (Table 1). Therefore, these results indicated that missing 3 ' splice site at the boundary of intron 3 and exon 4 resulted in exon 4 skipping of the mutant samples. In the 3' splice site deleted mutants, exon 4 was skipped; exons 3 and exon 5 were inappropriately combined.

\section{Deletion of 22 bp nucleotides in exon 4 resulted in stochastic skipping of exon 4}

We examined whether the lacking mutant 22 bp nucleotides in exon 4 also caused exon 4 skipping. We amplified 


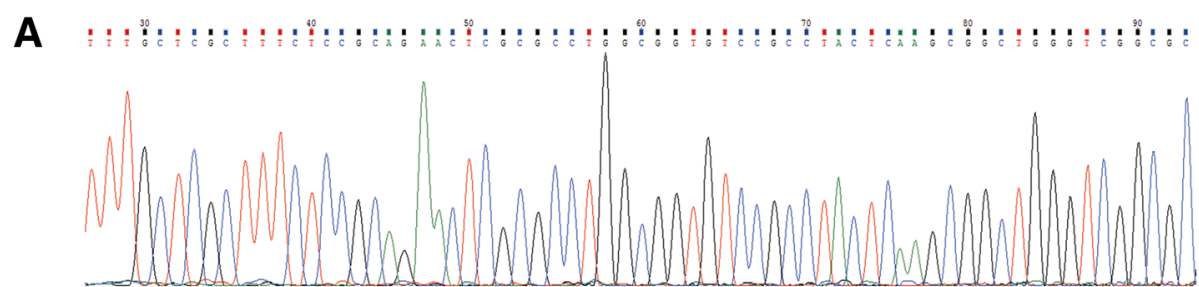

B

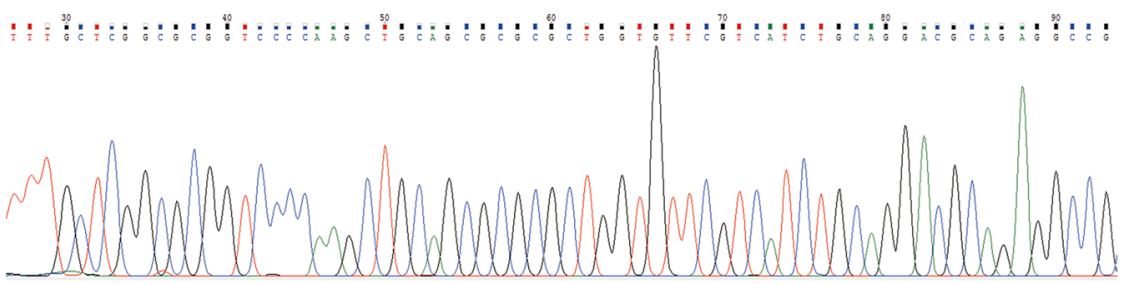

C TTtgCtcGCTTtCTCCGCAGAACTCGCGCCTGGCGGTGTCCGCCTACTCAAGC GGCTGGGTCGGCGCGGTCCCCCAGCTGCAGCGCGCGCTGGTGTTCGTCATCT GCAGGACGCAGAGGCCGCTGGGGCTGACCGCTGGTAAATTCTACTACGTCTC GCGGGAGTCGTTTGTCAGC

\section{TTtGCtCGgCGCGGTCCCCCAGCTGCAGCGCGCGCTGGTGTTCGTCATCT GCAGGACGCAGAGGCCGCTGGGGCTGACCGCTGGTAAATTCTACTACGTCTC GCGGGAGTCGTTTGTCAGC}

Fig. 2 Genome PCR products sequencing of the wildtype and the 55 bp mutant locust LmigOr35. a and $\mathbf{c}$ is the wildtype partial locust $L m i g O r 35$ gene sequencing trace and nucleotides sequence. Nucleotides marked with red only exist in the wild type locusts but not in the 55 bp mutant locusts. $\mathbf{b}$ and $\mathbf{d}$ is the $55 \mathrm{bp}$ mutant partial locust LmigOr35 gene sequencing trace and nucleotides sequence

the targeted sequences using PCR and run the gel. The 22 bp nucleotide deletion mutants had a smaller band (Fig. 4a). The sequencing of small band PCR products showed that the $22 \mathrm{bp}$ nucleotide deletion mutants lacked 22 bp nucleotides in the exon 4 of LmigOr35 (Fig. 4b). We extracted the total RNA from the antenna of the WT and $22 \mathrm{bp}$ nucleotide deletion mutant of the locusts. The total RNA was reversed into cDNA and amplified exon 2 to exon 5 of LmigOr35 using primers F2 and R2. We then run the gel and found that there was only one band in the wild type lines, however, there was two bands in $22 \mathrm{bp}$-nucleotides-deletion mutants (Fig. 4c). After the sequencing of PCR products, we determined that between the two bands in $22 \mathrm{bp}$ nucleotide missing mutants, the first band missed 22 bp nucleotides in exon 4 and the second band missed the entire exon 4 sequences in the 22 bp mutant cDNA products compared with the WT (Fig. 4d). Then, we cloned the PCR products and determined that approximately $26.72 \%$ of clones lacked exon 4 (Table 1). These results indicated that the missing 22 bp nucleotides in exon 4 of LmigOr35 resulted in stochastic exon 4 skipping.

\section{Discussion}

Our study shows that genome editing could unintendedly result in exon skipping in the migratory locust. Inducing indels in the exon can lead to stochastic exon skipping, whereas the deletion of the boundary of intron and exon ( 3 ' splice site) can cause the complete exon skipping. These findings prove that complete and stochastic exon skipping can result from the CRISPRmediated genome editing by deleting different regions of the gene.

Our study showed that the deletion of $22 \mathrm{bp}$ nucleotides resulted in stochastic exon 4 skipping in the exon 4 of LmigOr35. Approximately 26\% of mRNA displayed the lack of exon 4 in the $22 \mathrm{bp}$ mutant line of the migratory locust. Several recent studies have reported this stochastic exon skipping phenomenon caused by CRISPR-mediated editing of human FLOT1 and Ctnnb1 genes in culture cells [13, 15]. In fact, different kinds of point mutations, such as nonsense, missense, and translationally silent mutations, contributed to exon skipping [28-31]. Apart from the splice sites at the intron-exon boundaries, exons also 


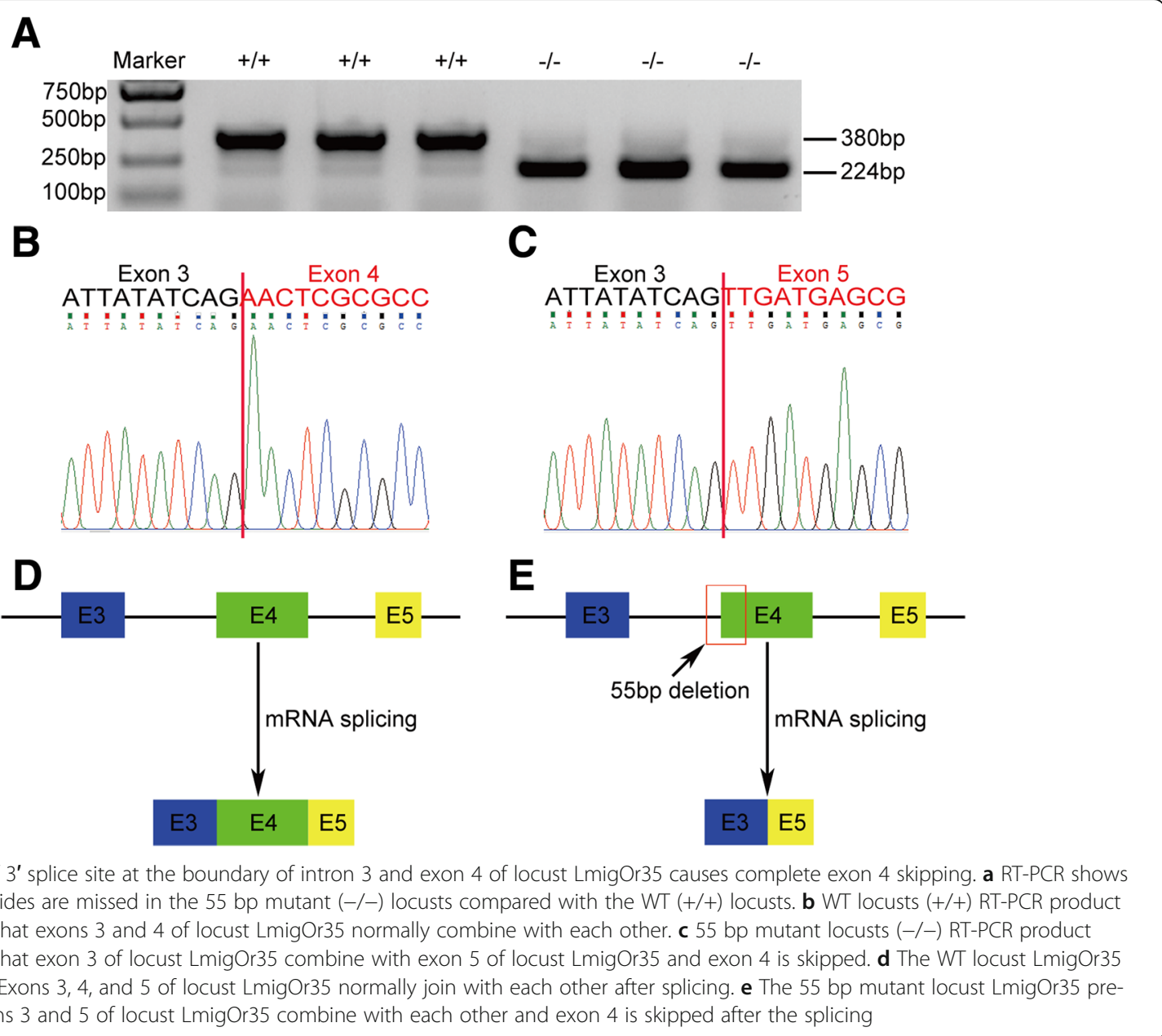

contained splicing elements, such as exon splicing enhancers, bind factors, and exon splicing silencers. Exon skipping caused by indels was because several indel mutations disrupted cis-acting sequences that promoted splicing $[16,28]$.

We determined that the boundary deletion of intron 3 and exon 4 ( 3 ' splice site) of LmigOr 35 by CRISPRmediated editing resulted in complete exon 4 skipping. Other previous studies showed that the frequency in which CRISPR-induced indels caused exon skipping was difficult to predict $[13,15]$. Our findings suggested that complete exon skipping was induced by CRISPRinduced indels at the boundary of intron and exon.
The indel caused by CRISPR-mediated editing resulted in the deletion of the $3^{\prime}$ splice site located at the boundary of intron 3 and exon 4 of LmigOr35. The 3' $\mathrm{OH}$ of exon 3 was unable to recognize the $3^{\prime}$ splice site located at the boundary of intron 3 and exon 4 . Therefore, the 3' $\mathrm{OH}$ of exon 3 recognized the next 3' splice site located at the boundary of intron 4 and exon 5 by attacking the $3^{\prime}$ splice site and separating intron 4 from exon 5. Then, intron 3, exon 4, and intron 4 were released from the pre-mRNA and exon 3 was combined with exon 5 that caused exon 4 skipping (Fig. 5). However, future work should be conducted to prove this hypothesis.

Table 1 The percent of exon skipping in WT, 22 bp mutant and 55 bp mutant locusts

\begin{tabular}{llll}
\hline & Analyzed clones (mean/SE) & Exon skipping clones(mean/SE) & Percentage of exon skipping clones(mean/SE) \\
\hline WT & $29 / 0.58$ & $0 / 0$ & $0 / 0$ \\
22 bp mutant & $27 / 2.52$ & $7 / 1.53$ & $26.72 / 0.07$ \\
55 bp mutant & $28 / 1.20$ & $28 / 1.20$ & $100 / 0$ \\
\hline
\end{tabular}



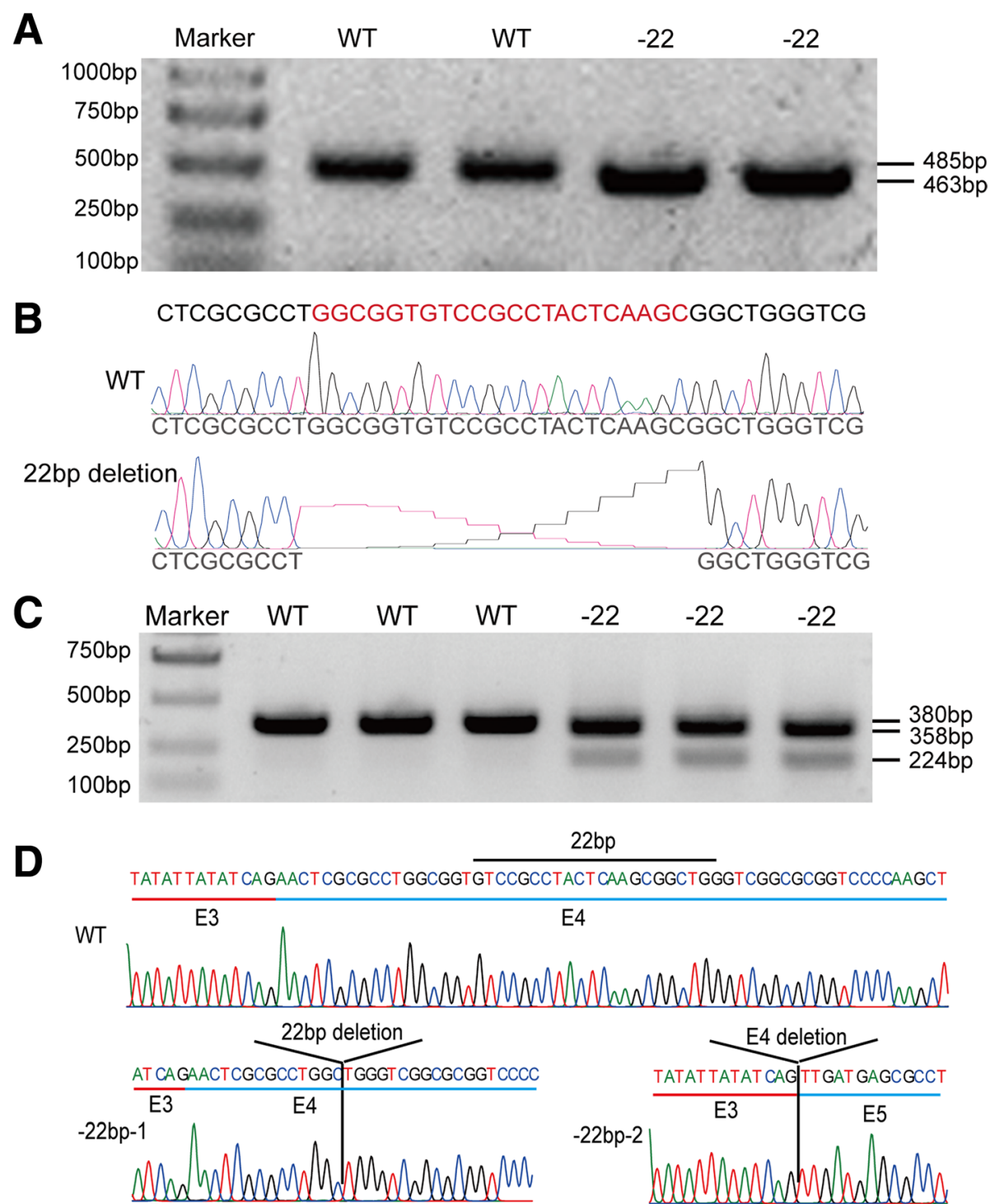

Fig. 4 Deleted 22 bp nucleotides in exon 4 of locust LmigOr35 result in alternative splicing and stochastic exon 4 skipping. a Genotype of WT and 22 bp mutant (-22) locusts. The WT locusts obtain a 485 bp brand and the -22 bp mutants obtain a 463 bp brand. b PCR product sequencing of the WT and - 22 bp mutant locust LmigOr35 shows that 22 bp nucleotide is missed in the exon 4 of -22 bp mutant. c RT-PCR shows that WT only has a 380 bp brand and -22 bp mutants have two brands, namely, the 358 bp and 224 bp brands. d RTPCR product sequencing of the WT and -22 bp mutant locust LmigOr35. In the WT locust LmigOr35 cDNA, exon 3 normally combines with exon 4. In the -22 bp mutant locust LmigOr35 cDNA, exon 3 combines with exon 4 (without the -22 bp nucleotides) in 358 bp brand $(-22 \mathrm{bp}-1)$, whereas exon 3 combines with exon 5 and exon 4 is skipped in the 224 bp brand $(-22$ bp -2$)$

\section{Conclusions}

Our studies revealed the effects of CRISPR-mediated editing on complete or stochastic exon skipping by deleting different regions in vivo. Combined with the results from other studies on cell lines, our observations suggested that CRISPR-mediated editing led to various splicing patterns that depended on the involved splicing regulatory elements. Although exon skipping was the unexpected consequence of CRISPR-mediated editing, it produced mRNA that encoded gain-of-function or partially functional proteins [15]. Currently, the effect of a given indel in exon on pre-mRNA splicing based on genomic sequences was difficult to predict because pre-mRNA splicing regulation was complicated. Thus, we should consider the posttranscript effects when establishing mutant organisms by CRISPR-mediated editing. Moreover, exon skipping caused by CRISPR-mediated editing might be a promising means to investigate the specific exon function by deleting the exon or to treat several genetic diseases caused by splicing mutations [32]. 

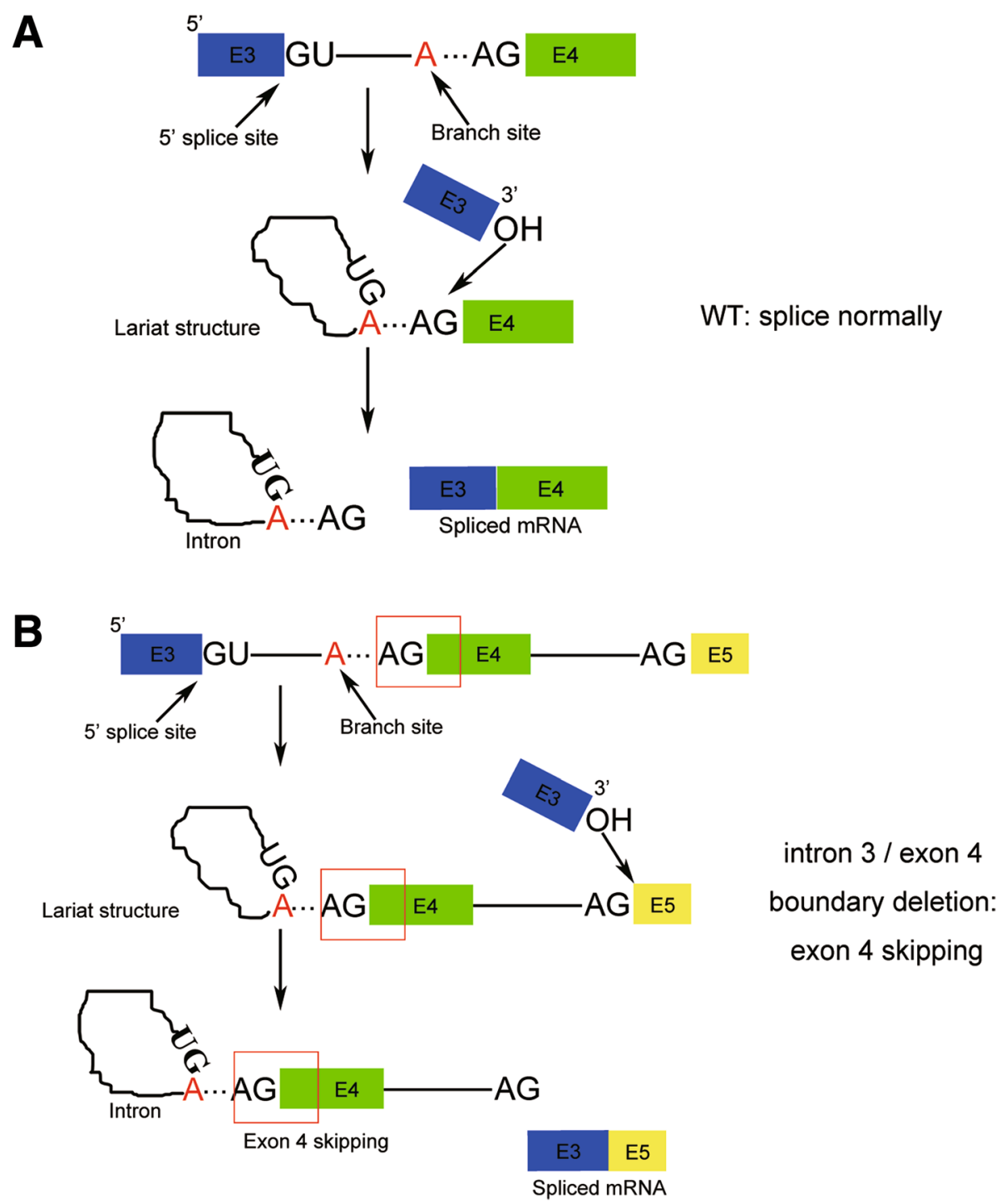

Fig. 5 Diagram of the WT and - 55 bp mutant locust LmigOr35 pre-mRNA splicing process. a In the WT locust, the branch site attacks the $5^{\prime}$ splice site and the lariat structure is formed. Then, $3^{\prime} \mathrm{OH}$ of exon 3 attacks the $3^{\prime}$ splice site by separating intron 3 from exon 4 and combining exon 3 with exon 4. Therefore, intron 3 of LmigOr35 is cut from the pre-mRNA, and exons 3 and 4 combine with each other. $\mathbf{b}$ In the -55 bp mutant locust, the lariat structure is normally formed, but the $3^{\prime}$ splice site is disrupted due to the deletion of 55 bp nucleotides. Therefore, $3^{\prime} \mathrm{OH}$ of exon 3 attacks the next 3' splice site located at the boundary of intron 4 and exon 5 . Intron 3, exon 4, and intron 4 are cut from the pre-mRNA, and exon 3 combines with exon 5. Exon 4 is skipped during the pre-mRNA splicing. A: the branch site, it locates in the 18-25 bp upstream of the 3' splice site and not includes in the 55 bp deleted nucleotides

\section{Methods}

\section{Insects}

The locusts used in the experiments were obtained from the breeding stock of Locusta migratoria at the Institute of Zoology, CAS, China. All locusts were reared under a 14:10 light/dark photo regime at $30 \pm 2{ }^{\circ} \mathrm{C}$ and were fed on a diet of fresh greenhouse-grown wheat seedlings and wheat bran.

\section{Generation of mutant locusts using CRISPR/Cas9 system}

The protocol to generate mutant locusts using CRISPR/ Cas9 system was previously described [7]. The embryos of locusts were collected from egg pods, washed with $75 \%$ ethanol, and were placed on $1 \%$ agarose gel. The purified Cas9 protein (Invitrogen, A36496, Massachusetts, USA) and guide RNA were mixed to final concentrations of 400 and $150 \mathrm{ng} / \mu \mathrm{l}$, respectively $(13.8 \mathrm{~nL})$, and were injected in the embryos using a microinjection machine. Then, the embryos were placed in a $30{ }^{\circ} \mathrm{C}$ incubator for approximately 14 days until the locusts hatched. The first-instar nymphs were placed in the cages with 14-h-light and sufficient food. We collected part of adult legs and lysed them with a $45 \mu \mathrm{L}$ NAOH buffer 
$(50 \mathrm{mM})$ at $95{ }^{\circ} \mathrm{C}$ in a PCR machine for $30 \mathrm{~min}$ and added $5 \mu \mathrm{L}$ Tris- $\mathrm{HCL}(\mathrm{PH}=8.0,1 \mathrm{M})$. Then, we used a $2 \mu \mathrm{L}$ template to amplify the targeted fragments, and we sequenced the fragments to identify whether the mutants were generated. The used primers were designed in introns 3 and 4 of LmigOr35 as follows: LmigOr35 intron 3-For, GTAAGTTCAGCCTGCTGTAT; LmigOr35 intron 4-Rev, and GTTTCAGCTAGTAGTACGAC. A 485 bp product was obtained from the WT locust after PCR reaction.

\section{Total RNA extraction}

Total RNA was extracted from the antenna of WT and mutant locusts using a TRNzol Reagent (TIANGEN BIOTECH CO., DP405-2, Beijing, China) based on the manuscript description. First, we cut the antennas of one locust, placed the two antennas in a $1.5 \mathrm{~mL}$ centrifuge tube, and placed the tube in liquid nitrogen for $20 \mathrm{~s}$. Second, we removed the tube and rapidly ground the antennas with a grinding rod until the antennas achieved a powder form. Third, we added $500 \mu \mathrm{L}$ of TRNzol Reagent in the tube and thoroughly mixed and we placed the tube at room temperature for $2 \mathrm{~min}$. Fourth, we added $100 \mu \mathrm{L}$ of chloroform into the tube, thoroughly mixed the solution, and centrifuged it at $12,000 \mathrm{rpm}$ for $10 \mathrm{~min}$ at $4{ }^{\circ} \mathrm{C}$. Fifth, $300 \mu \mathrm{L}$ of supernatant was placed into a new tube, and $300 \mu \mathrm{L}$ of isopropyl alcohol was added into the tube and thoroughly mixed. Afterward, the tube was placed at $-20{ }^{\circ} \mathrm{C}$ for $12 \mathrm{~h}$. Sixth, the tube was centrifuged at 12,000 rpm and $4{ }^{\circ} \mathrm{C}$ for $10 \mathrm{~min}$, the supernatant was removed, and $1000 \mu \mathrm{L}$ of $75 \%$ ethanol was added (prepared with nuclease-free water) in the tube followed by thorough mixing. Seventh, the tube was centrifuged at 12,000 rpm and $4{ }^{\circ} \mathrm{C}$ for $5 \mathrm{~min}$, the supernatant was removed, and the tube was placed at room temperature for $3 \mathrm{~min}$ to dry the RNA precipitation. Eighth, $20 \mu \mathrm{L}$ of nuclease-free water was added in the tube to dissolve the RNA precipitation and the RNA concentration was measured with Nano-drop 2000.

\section{RT-PCR and sequencing}

RNA was reverse-transcribed with $5 \mathrm{X}$ All-In-One RT MasterMix (Applied Biological Materials Inc., G490, British Columbia, Canada) according to the manufacturer's protocols. First, the RNA templates and 5X All-In-One RT MasterMix were thawed on ice. The solution was gently and thoroughly mixed. Second, the reaction mixture was prepared in a PCR tube on ice (total RNA $2 \mu \mathrm{g}+5 \mathrm{X}$ All-In-One RT MasterMix $4 \mu \mathrm{L}+$ nuclease-free water to $20 \mu \mathrm{L}$ ). Third, the components were well-mixed and collected by brief centrifugation. Fourth, the tube was incubated in the PCR machine for the reaction (at $25{ }^{\circ} \mathrm{C}$ for $10 \mathrm{~min}$, at $42{ }^{\circ} \mathrm{C}$ for $50 \mathrm{~min}$ for cDNA synthesis, and at $85{ }^{\circ} \mathrm{C}$ for $5 \mathrm{~min}$ ). Fifth, the tube was placed on ice to terminate the reaction. The newly synthesized first-strand cDNA was suitable for immediate downstream applications or for long-term storage at $-20^{\circ} \mathrm{C}$.

For the PCR reaction, we used a $2 \mathrm{X}$ TsingKe Master Mix (TsingKe Biotech Co., TSE004, Beijing, China) according to the manufacturer's protocols. To verify the exon deletion, we use the primers $\mathrm{F} 2$ and $\mathrm{R} 2$, which were designed in LmigOr35 exon 2 and LmigOr35 exon 5, respectively. The primers sequence are as follows: F2: GTTCTCCTTCAGTTCTTGGG; R2: CATTTGTCATTCACCTGGCG. The WT locust obtained a $380 \mathrm{bp}$ product after PCR reaction. After the PCR reaction, the PCR products executed the gel or sequencing in Beijing TsingKe Biotech Co., Ltd.

\section{Additional file}

Additional file 1: Figure S1. Mutant types generated by CRISPR/Cas9 system in exon 4 of locust LmigOr35. WT, wild type; - number, number of nucleotides deletion; $\Delta$ number, number of nucleotides substitution; (number), detected number of locusts; green letter, target; yellow letter, PAM; red letter, nucleotides of substitution; gray letter, nucleotides of deletion. (PDF $965 \mathrm{~kb}$ )

\section{Abbreviations}

CRISPR/Cas9: Clustered Regularly Interspaced Short Palindromic Repeats/ CRISPR associated protein 9; DSB: Double-strand breaks; Indels: Insertion-deletions; NHEJ: Non-homologous end joining; NMD: Nonsense-mediated mRNA decay; Or: Olfactory receptor; PCR: Polymerase chain reaction

\section{Funding}

L.K. is supported by the Strategic Priority Program of CAS (Grant NO. XDB11010000). X.W. is supported by the National Natural Science Foundation of China (Grant NO. 31772531). L.H is supported by the National Natural Science Foundation of China (Grant NO. 31601875).

\section{Availability of data and materials}

All data generated or analysed during this study are included in this published article [and its supplementary information files].

\section{Authors' contributions}

$D C, J X T, X W$ and LK designed the study. DC, JXT, BL, and LH performed experiments or analyzed data. DC, JXT, XW and LK wrote the manuscript with comments from all authors. All authors read and approved the final manuscript.

Ethics approval

Ethics approval was not needed for the study.

Consent for publication

Not applicable.

\section{Competing interests}

The authors declare that they have no competing interests.

\section{Publisher's Note}

Springer Nature remains neutral with regard to jurisdictional claims in published maps and institutional affiliations.

\section{Author details}

${ }^{1}$ State Key Laboratory of Integrated Management of Pest Insects and Rodents, Institute of Zoology, Chinese Academy of Sciences, Beijing 100101, China. ${ }^{2}$ Affiliated Hospital of Guangdong Medical University, Zhanjiang 
524001, China. ${ }^{3}$ University of Chinese Academy of Sciences, Beijing 100049, China.

Received: 13 June 2018 Accepted: 31 August 2018

Published online: 25 September 2018

\section{References}

1. Cong L, Ran FA, Cox D, Lin S, Barretto R, Habib N, et al. Multiplex genome engineering using CRISPR/Cas systems. Science. 2013;339:819-23.

2. Mali P, Yang L, Esvelt KM, Aach J, Guell M, DiCarlo JE, et al. RNA-guided human genome engineering via Cas9. Science. 2013;339:823-6.

3. Wang H, Yang H, Shivalila CS, Dawlaty MM, Cheng AW, Zhang F, et al. Onestep generation of mice carrying mutations in multiple genes by CRISPR/ Cas-mediated genome engineering. Cell. 2013;153:910-8.

4. Li W, Teng F, Li T, Zhou Q. Simultaneous generation and germline transmission of multiple gene mutations in rat using CRISPR-Cas systems. Nat Biotechnol. 2013;31:684-6.

5. Hwang WY, Fu Y, Reyon D, Maeder ML, Tsai SQ, Sander JD, et al. Efficient genome editing in zebrafish using a CRISPR-Cas system. Nat Biotechnol. 2013:31:227-9.

6. Friedland AE, Tzur YB, Esvelt KM, Colaiácovo MP, Church GM, Calarco JA. Heritable genome editing in C. Elegans via a CRISPR-Cas9 system. Nat Methods. 2013;10:741-3.

7. Li Y, Zhang J, Chen D, Yang P, Jiang F, Wang X, et al. CRISPR/Cas9 in locusts: successful establishment of an olfactory deficiency line by targeting the mutagenesis of an odorant receptor co-receptor (Orco). Insect Biochem Mol Biol. 2016;79:27-35.

8. Shan Q, Wang Y, Li J, Zhang Y, Chen K, Liang Z, et al. Targeted genome modification of crop plants using a CRISPR-Cas system. Nat Biotechnol. 2013:31:686-8.

9. Liang P, Xu Y, Zhang X, Ding C, Huang R, Zhang Z, et al. CRISPR/Cas9mediated gene editing in human tripronuclear zygotes. Protein Cell. 2015;6:363-72.

10. Matera AG, Wang Z. A day in the life of the spliceosome. Nat Rev Mol Cell Biol. 2014;15:108-21.

11. Sharpe JJ, Cooper TA. Unexpected consequences: exon skipping caused by CRISPR-generated mutations. Genome Biol. 2017;18:109.

12. Uddin B, Chen NP, Panic M, Schiebel E. Genome editing through large insertion leads to the skipping of targeted exon. BMC Genomics. 2015 16:1082.

13. Kapahnke M, Banning A, Tikkanen R. Random splicing of several exons caused by a Single Base change in the target exon of CRISPR/Cas9 mediated gene knockout. Cell. 2016;5. pii:E45.

14. Lalonde S, Stone OA, Lessard S, Lavertu A, Desjardins J, Beaudoin M, et al. Frameshift indels introduced by genome editing can lead to in-frame exon skipping. PLoS One. 2017;12:e0178700

15. Mou H, Smith JL, Peng L, Yin H, Moore J, Zhang XO, et al. CRISPR/Cas9mediated genome editing induces exon skipping by alternative splicing or exon deletion. Genome Biol. 2017;18:108.

16. Prykhozhij SV, Steele SL, Razaghi B, Berman JN. A rapid and effective method for screening, sequencing and reporter verification of engineered frameshift mutations in zebrafish. Dis Model Mech. 2017;10:811-22.

17. Black DL. Mechanisms of alternative pre-messenger RNA splicing. Annu Rev Biochem. 2003;72:291-336.

18. Wang $X$, Kang L. Molecular mechanisms of phase change in locusts. Annu Rev Entomol. 2014;59:225-44.

19. Wang Y, Yang P, Cui F, Kang L. Altered immunity in crowded locust reduced fungal (Metarhizium anisopliae) pathogenesis. PLoS Pathog. 2013;9:e1003102.

20. Wu R, Wu Z, Wang X, Yang P, Yu D, Zhao C, et al. Metabolomic analysis reveals that carnitines are key regulatory metabolites in phase transition of the locusts. Proc Natl Acad Sci U S A. 2012;109:3259-63.

21. Ma Z, Guo W, Guo X, Wang X, Kang L. Modulation of behavioral phase changes of the migratory locust by the catecholamine metabolic pathway. Proc Natl Acad Sci U S A. 2011;108:3882-7.

22. Guo W, Wang X, Ma Z, Xue L, Han J, Yu D, et al. CSP and takeout genes modulate the switch between attraction and repulsion during behavioral phase change in the migratory locust. PLoS Genet. 2011;7:e1001291.

23. Hou L, Yang P, Jiang F, Liu Q, Wang X, Kang L. The neuropeptide F/nitric oxide pathway is essential for shaping locomotor plasticity underlying locust phase transition. Elife. 2017;6. pii:e22526.
24. He J, Chen Q, Wei Y, Jiang F, Yang M, Hao S, et al. MicroRNA-276 promotes egg-hatching synchrony by up-regulating brm in locusts. Proc Natl Acad Sci U S A. 2016;113:584-9.

25. Wang $X$, Fang $X$, Yang $P$, Jiang $X$, Jiang $F$, Zhao $D$, et al. The locust genome provides insight into swarm formation and long-distance flight. Nat Commun. 2014:5:2957.

26. Shepard S, McCreary M, Fedorov A. The peculiarities of large intron splicing in animals. PLoS One. 2009;4: e7853.

27. Wang $Z$, Yang $P$, Chen D, Jiang F, Li Y, Wang $X$, et al. Identification and functional analysis of olfactory receptor family reveal unusual characteristics of the olfactory system in the migratory locust. Cell Mol Life Sci. 2015;72:4429-43.

28. Liu HX, Cartegni L, Zhang MQ, Krainer AR. A mechanism for exon skipping caused by nonsense or missense mutations in BRCA1 and other genes. Nat Genet. 2001;27:55-8.

29. Cartegni L, Chew SL, Krainer AR. Listening to silence and understanding nonsense: exonic mutations that affect splicing. Nat Rev Genet. 2002; 3:285-98.

30. Wang Z, Rolish ME, Yeo G, Tung V, Mawson M, Burge CB. Systematic identification and analysis of exonic splicing silencers. Cell. 2004;119:831-45.

31. Singh G, Pratt G, Yeo GW, Moore MJ. The clothes make the mRNA: past and present trends in mRNP fashion. Annu Rev Biochem. 2015;84:325-54.

32. Burghes AH, McGovern VL. Antisense oligonucleotides and spinal muscular atrophy: skipping along. Genes Dev. 2010;24:1574-9.

\section{Ready to submit your research? Choose BMC and benefit from:}

- fast, convenient online submission

- thorough peer review by experienced researchers in your field

- rapid publication on acceptance

- support for research data, including large and complex data types

- gold Open Access which fosters wider collaboration and increased citations

- maximum visibility for your research: over $100 \mathrm{M}$ website views per year

At BMC, research is always in progress.

Learn more biomedcentral.com/submissions 